\title{
Zur Ausdehnung der Spinwellentheorie der Ferromagnetica auf höhere Temperaturen
}

\author{
Von G. Heber \\ Aus dem Theoretisch-Physikalischen Institut der Universität Jena \\ (Z. Naturforschg. 8a, 91-97 [1953]; eingegangen am 5. Oktober 1953)
}

\begin{abstract}
Es wird versucht, einige Näherungsannahmen zu beseitigen, welche die Beschränkung der bekannten Spinwellentheorie auf tiefe Temperaturen zur Folge haben. Es handelt sich um eine bessere Näherung bei der Bestimmung der Eigenwerte der Energie und einige Verbesserungen bei der statistischen Auswertung des Energiespektrums. Am Beispiel der spontanen Magnetisierung sieht man, daß tatsächlich eine bessere Temperaturabhängigkeit dieser Größe gewonnen ist, was zu der Hoffnung berechtigen dürfte, die hier vorgelegte Verallgemeinerung der Spinwellentheorie sei auch in anderer Hinsicht bei höheren Temperaturen brauchbarer als die ältere Form der Theorie.
\end{abstract}

$\mathrm{E}$ $\mathrm{s}$ ist allgemein bekannt, daß die Spinwellentheorie der Ferromagnetica nur bei ,tiefen Temperaturen" (relativ zum Curie-Punkte) eine gute Näherung darstellt. Aber über die Größe des Temperaturintervalles, innerhalb dessen die genannte Theorie eine vernünftige Approximation an die Wirklichkeit darstellen dürfte, gehen die Meinungen weit auseinander. Während z. B. Wohlfarth $^{1}$ die Anwendbarkeit der Methode oberhalb $10^{-30} \mathrm{~K}$ bezweifelt, glauben andere Autoren an eine Gültigkeit der Theorie bis etwa $100^{\circ} \mathrm{K}$, wie es durch die experimentellen Untersuchungen über die Temperaturabhängigkeit der spontanen Magnetisierung einiger Ferromagnetica von $\mathrm{Fallot}^{2}$ nahegelegt wird.

Eine Klärung dieser strittigen Frage ist in mancher Hinsicht wünschenswert; sie ist wohl am einfachsten in der Form möglich, daß man die Punkte der Theorie aufsucht und zu beseitigen sucht, welche die Beschränkung der Methode auf tiefe Temperaturen zur Folge haben.

Hat man so eine allgemeinere Theorie geschaffen, so erkennt man durch Vergleich mit der spezielleren den Bereich der genäherten Übereinstimmung, d. h. das Gültigkeitsintervall der älteren Theorie. Selbstverständlich ist eine Ausdehnung der Spinwellentheorie auf höhere Temperaturen auch ganz unabhängig von der Frage nach der Abgrenzung der älteren Theorie sehr erwünscht.

Diese Überlegungen haben zu der vorliegenden Untersuchung geführt, in welcher einige wesentliche Vernachlässigungen der genannten älteren

1 E. P. Wohlfarth, Proc. Leeds philos. lit. Sec., sci. Sect. 5, 213 [1949].
Theorie nach Möglichkeit beseitigt werden. Es handelt sich um folgende Punkte:

1. Die Energieniveaus des Kristalles bei Vorhandensein von mehr als einem umgeklappten Spin werden etwas genauer bestimmt als bisher.

2. In der statistischen Berechnung makroskopischer Größen wird die Summation nicht mehr bis $\mathrm{zu}$ unendlich großen Spinwellenenergien durchgeführt, sondern nur noch bis zu einer endlichen Maximalenergie, entsprechend der endlichen Breite des Spinwellenbandes. Außerdem wird hier selbstverständlich die unter 1. erwähnte genauere Kenntnis der Energieniveaus verwendet.

Nicht diskutiert wird der Einfluß der Nichtorthogonalität der atomaren Eigenfunktionen, auf welchen Slater ${ }^{3}$ kürzlich hingewiessn hat. - Eine Berücksichtigung derWechselwirkung mit übernächsten Nachbaratomen im Gitter wäre wohl ohne gröBere Schwierigkeiten möglich; wir verzichten aber darauf, um die Methode möglichst klar darlegen zu können. Der Einfluß der unter 1. und 2. genannten Verbesserungen der Methode wird am Beispiel der Temperaturabhängigkeit der spontanen Magnetisierung $M(T)$ demonstriert und mit der Erfahrung verglichen

\section{$\S 1$. Genauere Bestimmung der Energie- Eigenwerte}

Wir gehen aus von dem bekannten Operator der Austauschenergie:

$$
H_{\mathrm{A}}=-2 \sum_{\text {Paare }} J_{l m} \Im_{l} \cdot \Im_{m}
$$

2 M. Fallot, Ann. Physique 6, 305 [1936].

3 J. C. Slater, Rev, mod. Physics 25, 199 [1953]. 
$\left(\Im_{l} \cdot \hbar=\right.$ Spinvektor-Operator des Atomes $l ; J_{l m}=$ Austauschintegral des Atompaares $l, m)$. Die $\Im_{l}$ genügen bekanntlich den Vertauschungsregeln:

$$
\Im_{l} \times \Im_{m}=i \delta_{l m} \Im_{l} .
$$

Mit Holstein und Primakoff ${ }^{4}$ führen wir zunächst für jedes $l$ statt der $x$ - und $y$-Komponenten der Operatoren $\mathfrak{\subseteq}_{l}, S_{l}^{x}$ und $\mathrm{S}_{l}^{y}$, Größen $S_{l}^{+}$und $S_{l}^{-}$ ein durch die Beziehungen

$$
S_{l}^{+}=S_{l}^{x}+i S_{l}^{y} ; \quad S_{l}^{-}=S_{l}^{x}-i S_{l}^{y} .
$$

Die Größen $S_{l}^{+}, S_{l}^{-}$und $S_{l}^{z}$ wieder werden dargestellt durch Operatoren $a_{l}, a_{l}^{*}$ gemäß den Definitionen

$$
\begin{aligned}
S_{l}^{+} & =(2 \sigma)^{1 / 2}\left(1-\frac{a_{l}^{*}}{2} \frac{a_{l}}{\sigma}\right)^{1 / 2} a_{l}, \\
S_{l}^{-} & =(2 \sigma)^{1 / 2} a_{l}^{*}\left(1-\frac{a_{l}^{*} a_{l}}{2 \sigma}\right)^{1 / 2}, \\
S_{l}^{z} & =\sigma-a_{l}^{*} a_{l} .
\end{aligned}
$$

Hier bedeutet $\sigma$ den größten Eigenwert von $S_{l}^{z}$, also die Größe des Spins eines Atomes des Kristalles in Einheiten $\hbar$; die Wurzeln aus Operatoren in (4) sind so $\mathrm{zu}$ verstehen, daß

$$
\left(1-\frac{a^{*} a}{2 \sigma}\right)^{1 / 2} \Phi(n)=\left(1-\frac{n}{2 \sigma}\right)^{1 / 2} \Phi(n),
$$

wenn $\quad a^{*} a \Phi(n)=n \Phi(n)$.

Aus (4), (3) und (2) folgen die Vertauschungsregeln für die $a, a^{*}$ :

$$
a_{l} a_{m}^{*}-a_{m}^{*} a_{l}=\delta_{l m}
$$

andere Kommutatoren verschwinden. Diese einfachen Regeln (5) sind es, derentwegen man die Operatoren $a, a^{*}$ eingeführt hat. Mit ihnen erhält (1) die Form

$$
\begin{array}{r}
H_{A}=-2 \sigma \sum_{\text {Paare }} J_{l m}\left[f_{l} a_{l} a_{m}^{*} f_{m}+a_{l}^{*} f_{l} f_{m} a_{m}\right. \\
\left.+\sigma-a_{l}^{*} a_{l} f_{m}^{2}-a_{m}^{*} a_{m} f_{l}{ }^{2}\right],
\end{array}
$$

wobei zur Abkürzung $f_{j}=\left(1-\frac{a_{j}^{*} a_{j}}{2 \sigma}\right)^{1 / 2}$ gesetzt ist. Die Diagonalisierung von (6) ist in Strenge offenbar sehr kompliziert; man kann jedoch für tiefe Temperaturen eine physikalisch plausible Näherung einführen und das genäherte $H_{\mathrm{A}}$ dann exakt diagonalisieren. Erinnern wir uns der Tatsache, daß $a_{j}{ }^{*} a_{j}$ gemäß Definition der Operator der Abweichung der $z$-Komponente des Spins des Atomes $j$ vom Maximalwerte $\sigma$ ist. Der Zustand der Sättigung des Fer-

4 T. Holstein u. H. Primak off, Physic. Rev, 58, $1098[1940]$. romagneticums am absoluten Temperatur-Nullpunkt ist dann dadurch gekennzeichnet, daß alle $a_{j}{ }^{*} a_{j}$ die Eigenwerte Null besitzen. Die $f_{j}$ sind dann also sämtlich gleich eins. Für etwas höhere Temperaturen werden auch einige höhere Eigenwerte von $a_{j}{ }^{*} a_{j}$ besetzt, entsprechend der Abnahme der spontanen Magnetisierung mit steigender Temperatur. Aber für tiefe Temperaturen wird die Abweichung $\operatorname{der} f_{j}$ von 1 noch nicht bedeutend sein; wir setzen genähert für tiefe Temperaturen einfach $f_{j}=1$.

In dieser Näherung läßt sich der Hamilton-Operator bekanntlich exakt diagonalisieren und liefert das Energie-Spektrum, in welchem sich die Energien der einzelnen Spinwellen ungestört überlagern. Wir deuten den Gang dieser Rechnung an. Ausgangspunkt ist also

$$
H_{\mathrm{A}}^{0}=-2 \sigma \sum_{\text {Paare }} J_{l m}\left[2 a_{l}^{*} a_{m}+\sigma-2 a_{l}^{*} a_{l}\right] .
$$

Durch einige Transformationen der Operatoren wird $H_{\mathrm{A}}^{0}$ als Summe von kommutierenden Termen dargestellt, deren jeder die Form des Hamilton-Operators eines harmonischen Oszillators besitzt. Da auch die Vertauschungsregeln mit denen eines harmonischen Oszillators übereinstimmen, sind die Eigenwerte damit bestimmt. -

Die erste der erwähnten Transformationen ist

$$
Q_{j}=\frac{1}{\sqrt{2}}\left(a_{j}+a_{j}^{*}\right) ; \quad P_{j}=\frac{1}{\sqrt{2} \cdot i}\left(a_{j}-a_{j}^{*}\right) .
$$

Die neuen Variablen befolgen die Vertauschungsregeln

$$
Q_{l} P_{j}-P_{j} Q_{l}=i \delta_{l j} .
$$

$H_{\mathrm{A}}^{0}$ erhält dann die Gestalt

$$
H_{\mathrm{A}}{ }^{0}=H_{1}{ }^{0}+H_{2}{ }^{0}+H_{3}{ }^{0},
$$

wobei $H_{1}{ }^{0}$ keine Operatoren mehr enthält:

$$
H_{1}^{0}=-2 \sigma \Sigma J_{l m}(\sigma+1),
$$

und weiter

$$
H_{2}{ }^{0}=+2 \sigma \Sigma J_{l m}\left(Q_{l}^{2}+P_{l}^{2}\right)
$$

$H_{3}{ }^{0}=-2 \sigma \Sigma J_{l m}\left[Q_{l} Q_{m}+P_{l} P_{m}+i\left(Q_{l} P_{m}-P_{l} Q_{m}\right)\right]$.

$\mathrm{H}_{2}{ }^{0}$ und $\mathrm{H}_{3}{ }^{0}$ müssen weiter behandelt werden. $\mathrm{Zu}$ diesem $Z$ weck führen wir eine Fourier-Transformation durch ${ }^{5}$

$Q_{l}=\frac{1}{\sqrt{N}} \sum_{k} e^{-i k l} Q_{k}, \quad P_{l}=\frac{1}{\sqrt{N}} \sum_{k} e^{+i k l} P_{k}$.

5 Die in der Summation zugelassenen $k$ sind wie üblich durch die Periodizitätsbedingung im Grundbereich festgelegt. $N=$ Zahl der Atome im Grundbereich. 
Die Rechnung wird vereinfacht, wenn wir an dieser Stelle die Annahme einführen, $J_{l m}$ sei nur für die $z$ nächsten Nachbarn eines Gitteratomes von Null verschieden und stets gleich $J$. Dann ergibt sich

$$
\begin{aligned}
& H_{2}{ }^{0}+H_{3}{ }^{0}=\sigma J \sum_{k}\left\{(z-\gamma(k))\left[Q_{-k} Q_{k}+P_{-k} P_{k}\right]\right. \\
& \left.-i \gamma(k)\left[Q_{-k} P_{-k}-Q_{k} P_{k}\right]\right\}
\end{aligned}
$$

mit $\gamma(k)=\sum_{\text {Nachb. }} e^{i k \varrho} ; \varrho=$ Vektor zum nächsten Nachbaratom. Da die Operatoren $Q_{k}, P_{k^{\prime}}$ mit $k \neq k^{\prime}$ kommutieren, sind nur noch die Summanden mit $+k$ und mit $-k$ vermischt; sie müssen noch getrennt werden. Diese beiden Summanden können wir wegen $\gamma(k)=\gamma(-k)$ zusammenfassen in

$$
H_{k}^{0}+H_{-k}^{0}=(z-\gamma(k))\left[Q_{k} Q_{-k}+P_{k} P_{-k}\right] .
$$

Die Separation gelingt mittels der Transformation $q_{k}^{+}=\frac{1}{\sqrt{2}}\left(Q_{k}+Q_{-k}\right) ; q_{k}^{-}=\frac{i}{\sqrt{2}}\left(P_{k}-P_{-k}\right) ;$ $p_{k}^{+}=\frac{1}{\sqrt{2}}\left(P_{k}+P_{-k}\right) ; p_{k}^{-}=\frac{i}{\sqrt{2}}\left(Q_{k}-Q_{-k}\right) ;$

sie ergibt nämlich $H_{k}^{0}+H_{-k}^{0}=(z-\gamma(k))\left[p_{k}^{+2}+{q_{k}^{+}}^{2}+p_{k}^{-2}+q_{k}^{-2}\right]$. Die $q_{k}^{+}, p_{k}^{+}$und $q_{k}^{-}, p_{k}^{-}$sind kanonisch konjugierte Paare mit den entsprechenden Vertauschungsregeln; der in Rede stehende Term hat deshalb die Eigenwerte

$2(z-\gamma(k))\left[n_{k}^{+}+n_{k}^{-}+1\right] ; n_{k}^{+}, n_{k}^{-}=0,1,2,3 \ldots$

Insgesamt erhalten wir also die Eigenwerte

$$
\begin{aligned}
E_{\mathrm{A}}^{0} & =H_{1}^{0}+2 \sigma J \sum_{|k|>0}(z-\gamma(k))\left[n_{k}^{+}+n_{k}^{-}+1\right] \quad(11) \\
& =\sigma J N z(\sigma+1)+2 \sigma J \sum_{k}(z-\gamma(k))\left(n_{k}+1 / 2\right) \\
& =\sigma^{2} J N z+2 \sigma J \sum_{k}(z-\gamma(k)) n_{k} ; n_{k}=0,1,2,3 \ldots
\end{aligned}
$$

(11) ist genau die Formel, welche schon Bloch in seiner Theorie der Spinwellen erhalten hatte; sie ist sicher unbrauchbar in der Nähe des Curie-Punktes, wo die $z$-Komponente des Spins beinahe jedes zweiten Atomes $-\sigma$ sein muß, also die $f_{l} \neq 1$ sind. Um in diesem Gebiet höherer Temperaturen eine bessere Näherung als (11) zu finden, erinnern wir uns der physikalischen Bedeutung der $f_{l}$. Die Operatoren $S_{l}^{+}, S_{l}^{-}$sind ja als Operatoren der Erzeugung bzw. Vernichtung der Einheit $\hbar$ der $z$-Komponente des Spins des Atomes $j$ zu deuten; $a_{l}$ und $a_{l}{ }^{*}$ sind ebenfalls Erzeugungs- bzw. Vernichtungsoperatoren, wie man aus (5) ersieht. Die Faktoren in (4) bringen zum Ausdruck, daß zu jedem Atom ein Maximalund ein Minimalwert $+\sigma$ und $-\sigma$ der $z$-Komponente des Spins gehört, daß also die Erzeugung bzw. Vernichtung von Spinkomponenten nicht beliebig weit getrieben werden kann. Diese Aufgabe der Operatoren kann aber auch von den gewöhnlichen Funktionen $f_{l}=\left(1-\frac{n_{l}}{2 \sigma}\right)^{1 / 2}$ übernommen werden, solange man nur mit Eigenfunktionen der $z$-Komponente des Spins der Atome $l$ arbeitet. $n_{l}$ ist dann der betreffende Eigenwert von $a_{l}{ }^{*} a_{l}$.

Unter der Annahme, alle $f_{l}$ seien in dieser Weise gewöhnliche Funktionen, keine Operatoren mehr, läßt sich $H_{\mathrm{A}}$ ebenfalls exakt diagonalisieren. Man muß sich allerdings darüber im klaren sein, daß man auf diese Weise nicht die exakten Eigenwerte von (1) gefunden hat, denn (1) kommutiert nicht mit den $S_{l}^{z}$, die beiden Operatoren lassen sich mithin in Strenge nicht gleichzeitig auf Diagonalform bringen. Wir dürfen jedoch hoffen, auf diese Weise eine einigermaßen brauchbare Näherung für die höheren Temperaturen zu erhalten.

Wir diagonalisieren also jetzt unter der eben formulierten Annahme den Operator

$$
H_{\mathrm{A}}^{1}=-2 \sigma \sum_{\text {Paare }} J_{l m}\left[2 f_{l} f_{m} a_{l}^{*} a_{m}+\sigma-f_{m}^{2} a_{l}^{*} a_{l}\right] .
$$

Die Rechnungen verlaufen weitgehend parallel den zur Diagonalisierung von $H_{\mathrm{A}}^{0}$ notwendigen. Zunächst wird wieder die Transformation (8) ausgeführt; sie liefert

$$
H_{\mathrm{A}}^{1}=H_{1}{ }^{1}+H_{2}{ }^{1}+H_{3}{ }^{1}
$$

mit

$$
\begin{aligned}
H_{1}{ }^{1}= & -2 \sigma \Sigma J_{l m}\left(\sigma+f_{m}{ }^{2}\right), \\
H_{2}{ }^{1}= & +2 \sigma \sum J_{l m}\left(Q_{l}{ }^{2}+P_{l}{ }^{2}\right) f_{m}{ }^{2}, \\
H_{3}{ }^{1}= & -2 \sigma \sum J_{l m}\left[Q_{l} Q_{m}+P_{l} P_{m}\right. \\
& \left.+i\left(Q_{l} P_{m}-P_{l} Q_{m}\right)\right] f_{l} f_{m} .
\end{aligned}
$$

Man beachte, daß $H_{1}{ }^{1}$ jetzt zwar keine Operatoren mehr enthält, aber über die $f_{l}$ doch noch vom Gesamtspin abhängt. - Weiter erhält man nach der Transformation (9) das Resultat

$$
\begin{aligned}
H_{2}{ }^{1}+H_{3}{ }^{1}=\sigma & \sum_{k}\left\{(\overline{z J}-\overline{J \gamma(k)})\left[Q_{-k} Q_{k}+P_{-k} P_{k}\right]\right. \\
& \left.-i \overline{J \gamma(k)}\left[Q_{-k} P_{-k}-Q_{k} P_{k}\right]\right\} .
\end{aligned}
$$

In (14) sind die Abkürzungen

$$
\overline{z J}=\Sigma J_{l m} f_{m}{ }^{2} ; \quad \overline{J_{\gamma}(k)}=\Sigma J_{l m} f_{l} f_{m} e^{i k(m-l)}
$$

eingeführt, in welchen die Summen bei festem $l$ über die nächsten Nachbarn $m$ des Atomes $l$ zu erstrecken sind. Austauschintegrale zwischen ent- 
fernteren als nächsten Nachbarn werden wiederum vernachlässigt. Setzt man noch voraus ${ }^{6}$, daß $\overline{J_{\gamma(k)}}$ $=\overline{J \gamma(-k)}$, so vollendet (10) die Aufspaltung von $H_{\mathrm{A}}^{1}$ in Oszillatorterme und man erhält die Eigenwerte der Energie

$$
\begin{array}{r}
E_{\mathrm{A}}^{1}=H_{1}^{1}+2 \sigma \sum_{k}\left(\overline{z J}-\overline{J_{\gamma}(k)}\right)\left[n_{k}+\frac{1}{2}\right] ; \\
n_{k}=0,1,2 \ldots .
\end{array}
$$

Mit (15) läßt sich auch $H_{1}{ }^{1}$ vereinfachen; es ergibt $\operatorname{sich} H_{1}^{1}=-\sigma N(\sigma z J+\overline{z J})$.

Unter Vernachlässigung der Schwankungen der $f_{l}$ von Atom zu Atom erhalten wir ferner

$$
\overline{z J}=z J f_{0}{ }^{2} \text { und } \overline{J \gamma(k)}=J f_{0}{ }^{2} \gamma(k) \text {. }
$$

$f_{0}^{2}$ spielt dann die Rolle eines Mittelwertes der $f_{l}{ }^{2}$ über alle Atome und die Zeit. Man sieht, daß offenbar nur bei Vernachlässigung von Schwankungen der $f_{l}$ die obige Annahme $\overline{J \gamma(k)}=\overline{J \gamma(-k)}$ i. allg. berechtigt ist. In einer solchen ,statischen“ Näherung ergibt sich somit endgültig:

$$
\begin{aligned}
E_{\mathrm{A}}^{1}= & -2 \sigma J N z\left(\sigma+f_{0}^{2}\right) \\
& +2 \sigma J f_{0}^{2} \sum_{k}(z-\gamma(k))\left(n_{k}+1 / 2\right) \\
= & -\sigma^{2} J N z+2 \sigma J f_{0}^{2} \sum_{k}(z-\gamma(k)) n_{k} .
\end{aligned}
$$

Vergleicht man (17) mit (11), so erkennt man, daß die Nullpunktsenergie der Spinwellen (der für $n_{k}=0$ verbleibende Teil von $E_{\mathrm{A}}$ ) sich nicht geändert hat; lediglich in dem mit $n_{k}$ behafteten Teil der Energie ist $f_{0}^{2} J$ an die Stelle von $J$ getreten.

Was bedeutet diese Änderung für das Energiespektrum der Spinwelle? $-f_{0}^{2}$ war eingeführt worden als örtlicher und zeitlicher Mittelwert aller $f_{l}^{2}=\left(1-\frac{n_{l}}{2 \sigma}\right)$, wobei $n_{l}=$ Abweichung des Spins des Atomes $l$ vom Maximalwert $\sigma$ (in Einheiten $\hbar$ ). $\sigma-n_{l}=S_{l}$ ist aber ein Eigenwert der $z$-Komponente des Spins des Atomes $l$, also können wir auch schreiben

$f_{0}^{2}=\frac{1}{2 \sigma}(2 \sigma-\bar{n})=\frac{1}{2 \sigma}(\sigma+\bar{S})=\frac{1}{2}(1+m)$.

Hier sind $\bar{n}, \bar{S}$ die Mittelwerte von $n_{l}$ bzw. $S_{l}$; $m=\frac{\bar{S}}{\sigma}=\frac{M(T)}{M(0)}$ ist die relative spontane Magnetisierung unseres Kristalles bei der Temperatur $T$. $f_{0}^{2}$ ist also in der Nähe des absoluten Nullpunktes

${ }^{6}$ Die Berechtigung dieser Annahmewird weiter unten untersucht. der Temperatur etwa gleich 1 , in der Nähe der Curie-Temperatur $T_{\mathrm{c}}$ aber ungefähr gleich $1 / 2$. Unser Energiespektrum für die Spinwellen wird also temperaturabhängig und zwar sieht die Energiedichte $g(\varepsilon)$ im Spinwellenband bei den beiden charakteristischen Temperaturen $T=0$ und $T=T_{\mathrm{c}}$ schematisch wie in Abb. 1 gezeigt aus (die Flächen unter den beiden Kurven müssen gleich sein; es gibt ja immer genau $N$ Eigenwerte).

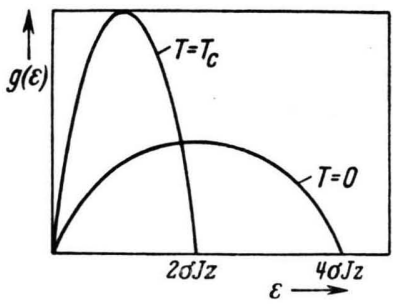

Abb. 1. Energiedichte $g$ über der Energie $\varepsilon$ der Spinwellen für zwei charakteristische Temperaturen (schematisiert).

Leider bekommt unsere Theorie durch die Abhängigkeit der Energiedichte von der Temperatur (bzw. der spontanen Magnetisierung) einen gewissen phänomenologischen Einschlag; doch scheint sich dieser Nachteil im Augenblick kaum auf einfache Weise umgehen zu lassen. Es sei übrigens schon hier erwähnt, daß wir die Funktion $m(T)$ nicht etwa aus irgendeiner anderen Theorie in die Gl. (17) einzuführen brauchen, sondern daß diese Größe zunächst als Parameter stehen bleibt und im nächsten Paragraphen innerhalb unserer Theorie auf statistischem Wege bestimmt wird.

\section{$\S 2$. Bestimmung der spontanen Magnetisierung}

Nachdem wir in $\S 1$ die erlaubten Energieniveaus für die Spinwellen gefunden haben, können wir uns nun der Bestimmung statistischer Mittelwerte makrophysikalischer Größen zuwenden. Da jedes Niveau durch beliebig viele Spinwellen besetzt werden kann, können die Spinwellen bekanntlich als ,,Teilchen" behandelt werden, welche der Bose-Statistik genügen. Die gewünschten Mittelwerte erhält man deshalb aus der Zustandssumme

$$
Z=\prod_{k} \frac{1}{1-e^{-\beta \varepsilon_{k}}},
$$

wobei $\beta=1 / k T$ und $\varepsilon_{k}$ die zur Wellenzahl $k$ gehörige Energie einer Spinwelle ist. [Man schreibe zur Definition der $\varepsilon_{k}(17)$ in der Form: $E_{\mathrm{A}}^{1}=-\sigma^{2} J N z+$ $\sum_{k} \varepsilon_{k} n_{k}$; also $\varepsilon_{k}=2 \sigma J f_{0}^{2}(z-\gamma(k))$.] Zu beachten ist noch, daß (18) für eine variable, unbeschränkte 
Zahl von Bose-Teilchen gilt; im Falle der Statistik der Spinwellen wäre es sachgemäßer, eine variable Teilchenzahl mit oberer Grenze $(=N / 2)$ einzuführen. Wir wollen jedoch im Augenblick diese Komplikation nicht ins Auge fassen und uns mit der einfacheren Gl. (18) begnügen.

Als Beispiel der Anwendung der Statistik und Möglichkeit zum Vergleich mit der Erfahrung befassen wir uns jetzt mit der Bestimmung der mittleren spontanen Magnetisierung $M$ bei bestimmter Temperatur $T$. Zu diesem Zwecke muß zunächst ein äußeres Magnetfeld $H$ eingeführt und $\varepsilon_{k}(H)$ ermittelt werden. $M(T)$ ergibt sich dann aus der bekannten Formel

$$
M(T)=\frac{1}{\beta} \cdot \frac{\partial}{\partial H}(\ln Z) .
$$

Wir überspringen diesen Teil der Überlegungen, da sie gegenüber der älteren Theorie nichts Neues enthalten, und geben sofort die Endformel für $M(T)$ an, in welcher $H$ wieder nach null streben darf

$M(T)=\mu\left(N-\sum_{k} \frac{1}{e^{\beta \varepsilon_{k}}-1}\right)=\mu\left(N-\sum_{\varepsilon} \frac{g(\varepsilon)}{e^{\beta \varepsilon}-1}\right)$.

Wir werden die letzte Fassung der Gl. (19) bevorzugen, in welcher $g(\varepsilon)$ die Zahl der Eigenwerte mit gleicher Energie $\varepsilon$ bedeutet. Das aus $\S 1$ folgende $g(\varepsilon)$ ist in Abb. 1 schematisch dargestellt worden. Leider bereitet die Auswertung von (19) mit einem $g(\varepsilon)$ von diesem Typ größere Schwierigkeiten, so daß wir wiederum gezwungen sind, genähert mit einfacheren $g \mathrm{zu}$ arbeiten. - Die folgenden Formeln gelten übrigens nur für das einfach-kubische Gitter; doch sind auch andere Gittertypen im Prinzip genau so zu behandeln. -

Die alte Blochsche Spinwellentheorie für tiefe Temperaturen arbeitete mit der Näherung

$$
g_{\mathrm{a}}(\varepsilon)=\frac{3}{2} N\left(2 \pi^{2} \sigma J\right)^{-3 / 2} \varepsilon^{1 / 2} .
$$

Dieses $g$ schmiegt sich für kleine $\varepsilon$ genau dem aus $\S 1$ mit $f_{0}^{2}=1$ erhaltenen $g(\varepsilon)$ an, gibt jedoch für große $\varepsilon$ einen völlig falschen Verlauf, indem es kein abgeschlossenes Band darstellt. Außerdem gibt (20 a) nicht die in $\S 1$ erhalteneTemperaturabhängigkeit von $g$ wieder. Achten wir zunächst nicht auf das Abbrechen von $g$ für große $\varepsilon$, so können wir die

${ }^{7}$ Nachdem wir, wie üblich, das Diskontinuum durch ein entsprechendes Kontinuum von erlaubten Zuständen ersetzt haben.
Temperaturabhängigkeit von $g$ für kleine $\varepsilon$ zutreffend darstellen durch

$$
g_{\mathrm{b}}(\varepsilon)=\frac{3}{2} N\left(2 \pi^{2} \sigma J f_{0}^{2}\right)^{-3 / 2} \varepsilon^{1 / 2} .
$$

Schließlich wollen wir das in Abb. 1 gezeigte $\mathrm{Ab}$ brechen des Energiespektrums idealisierend so beachten, daß wir mit

$$
\begin{aligned}
& g_{\mathrm{c}}(\varepsilon)=\left\{\begin{array}{cc}
g_{\mathrm{a}}(\varepsilon) & \text { für } \varepsilon<\varepsilon_{\mathrm{c}} \\
0 & \text { für } \varepsilon>\varepsilon_{\mathrm{c}}
\end{array}\right. \\
& g_{\mathrm{d}}(\varepsilon)=\left\{\begin{array}{cc}
g_{\mathrm{b}}(\varepsilon) & \text { für } \varepsilon<\varepsilon_{\mathrm{d}} \\
0 & \text { für } \varepsilon>\varepsilon_{\mathrm{d}}
\end{array}\right.
\end{aligned}
$$

arbeiten. $\varepsilon_{\mathbf{c}}$ und $\varepsilon_{\mathrm{d}}$ sind dabei so bestimmt, daß die Zahl der Eigenwerte genau gleich $N$ ist, d. h. $\varepsilon_{\mathrm{c}}=2 \pi^{2} \sigma J ; \varepsilon_{\mathrm{d}}=2 \pi^{2} \sigma J f_{0}{ }^{2}$. Wir wollen mit den vier verschiedenen Energiespektren $(20 \mathrm{a} . . \mathrm{d})$ die spontane Magnetisierung berechnen, um den Einfluß der verschiedenen Vernachlässigungen der alten Spinwellentheorie auf diese makroskopische Größe zu studieren. In allen vier Fällen (künftig als a ...d) bezeichnet) stoßen wir hierbei ${ }^{7}$ auf Integrale vom Typ

$$
I=c \int_{0}^{\varepsilon_{0}} \frac{\varepsilon^{1 / 2} \mathrm{~d} \varepsilon}{e^{\beta \varepsilon}-1}
$$

und zwar ist $\quad M=\mu(N-I)$;

ferner $\quad c= \begin{cases}\frac{3}{2} N\left(2 \pi^{2} \sigma J f_{0}^{2}\right)^{-3 / 2} & \text { im Falle e und } \mathrm{d}, \\ \frac{3}{2} N\left(2 \pi^{2} \sigma J\right)^{-3 / 2} & \text { im Falle a und } c,\end{cases}$

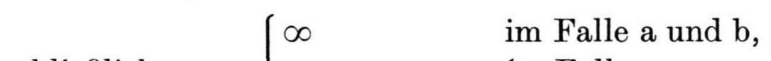
schließlich $\varepsilon_{0}= \begin{cases}\infty & \text { im Falle c, } \\ \varepsilon_{\mathrm{c}} & \text { im Falle d. }\end{cases}$

Wir integrieren (21), indem wir den Nenner in eine Reihe entwickeln und erhalten

$$
\begin{gathered}
I=c \int_{0}^{\varepsilon_{0}} \varepsilon^{1 / 2}\left(e^{-\beta \varepsilon}+e^{-2 \beta \varepsilon}+e^{-3 \beta \varepsilon}+\ldots\right) \mathrm{d} \varepsilon=c \sum_{n=1}^{\infty} \Im_{n} \\
\Im_{n}=\int_{0}^{\varepsilon_{0}} \varepsilon^{1 / 2} e^{-n \beta \varepsilon} \mathrm{d} \varepsilon=(n \beta)^{-3 / 2} \int_{0}^{n \beta \varepsilon_{0}} e^{-t} t^{1 / 2} \mathrm{~d} t .
\end{gathered}
$$

$\Im_{n}$ ist also bis auf den Faktor die tabuliert vorliegende ${ }^{8}$,Unvollständige Fakultät“", welche definiert werden kann durch das Integral

$$
(x, y) ! \equiv \int_{0}^{y} e^{-t} t^{x} \mathrm{~d} t
$$

\footnotetext{
${ }^{8}$ Pearson, Tables of the Incomplete $\Gamma$-Funktion, London 1922.
} 
Wir können also notieren

und

$$
\mathfrak{\Im}_{n}=(n \beta)^{-3 / 2}\left(\frac{1}{2}, n \beta \varepsilon_{0}\right) !
$$

$$
I=c \sum_{n=1}^{\infty}(n \beta)^{-3 / 2}\left(\frac{1}{2}, n \beta \varepsilon_{0}\right) !=c \beta^{-3 / 2} \eta(z) .
$$

Wir haben hier die Funktion $\eta(z)$ eingeführt, welche durch

$$
\eta(z)=\sum_{n=1}^{\infty} n^{-3 / 2}\left(\frac{1}{2}, n z\right) ! ; \quad z=\beta \varepsilon_{0}
$$

definiert ist und deren numerischer Ermittlung wir uns sogleich zuwenden müssen.

Zunächst bemerken wir, daß in unserem Falle a (23) natürlich das Blochsche $T^{3 / 2}$-Gesetz enthält, denn für $\varepsilon_{0} \rightarrow \infty, z \rightarrow \infty$ wird $(1 / 2, \infty) !=(1 / 2) !$ und $\eta(\infty)=(1 / 2) ! \zeta(3 / 2) ; \zeta(x)=$ Riemannsche $\zeta$-Funktion, also

$$
I_{\mathrm{a}}=\frac{3}{2} N\left(\frac{k T}{2 \pi^{2} \sigma J}\right)^{3 / 2}\left(\frac{1}{2}\right) ! \zeta\left(\frac{3}{2}\right) .
$$

Daneben können wir auch sofort das Resultat für den Fall b hinschreiben; es ist ersichtlich:

$$
I_{\mathrm{b}}=\frac{3}{2} N\left(\frac{k T}{2 \pi^{2} \sigma J f_{0}^{2}}\right)^{3 / 2}\left(\frac{1}{2}\right) ! \zeta\left(\frac{3}{2}\right) .
$$

Da $f_{0}^{2}$ noch gemäß (18) von $m(T)$ abhängt, erhält man im Falle b nur eine implizite Gleichung für $m(T)$, welche wir numerisch aufgelöst und in Abb. 2, Kurve b dargestellt haben. Kurve a in Abb. 2 ist das $T^{3 / 2}$-Gesetz gemäß unserem Falle a.

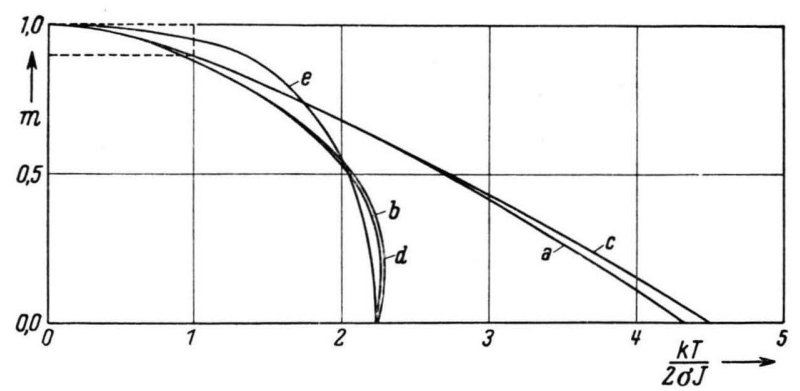

Abb. 2. Spontane Magnetisierung $m$ in Abhängigkeit von der Temperatur T. a, b, c, d theoretische Gesetze, e experimentelle Kurve.

Da die in (23) eingeführte Funktion $\eta(z)$ noch nicht tabuliert vorliegt, müssen wir uns zur Bestimmung von $m(T)$ in den Fällen $\mathrm{c}$ und d mit dieser Aufgabe befassen. Beachten wir, daß $\eta(\infty)=$ $(1 / 2) ! \zeta(3 / 2)$, so liegt es nahe, $\eta(z)$ in der Form zu schreiben $\eta(z)=(1 / 2) !\{\zeta(3 / 2)-\Delta(z)\}$, wo

$$
\Delta(z)=\sum_{n=1}^{\infty} n^{-3 / 2}\left[1-\frac{(1 / 2, n z) !}{(1 / 2, \infty) !}\right] .
$$

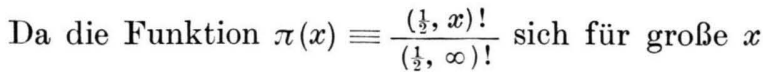
exponentiell dem Werte 1 nähert (schon für $x=5$ ist $\pi(x)=0,9934)$, werden für nicht zu kleine Werte von $z$ die höheren Glieder der Reihe sehr schnell exponentiell klein und es genügt, die ersten Glieder mitzunehmen, um $\Delta(z)$ mit für unsere Zwecke ausreichender Genauigkeit zu ermitteln. So ist ohne allzugroßen Aufwand Tab. 1 für $\Delta(z)$ entstanden.

\begin{tabular}{|c|c|c|c|c|c|}
\hline$z$ & 0 & 2 & 3 & 4 & 5 \\
\hline$\Delta(z)$ & 2,612 & 0,1870 & 0,0624 & 0,0205 & 0,0067 \\
\hline$z$ & 6 & 7 & 8 & 9 & 10 \\
\hline$\Delta(z)$ & $22 \cdot 10^{-4}$ & $7 \cdot 10^{-4}$ & $2 \cdot 10^{-4}$ & $<1 \cdot 10^{-4}$ & $<2 \cdot 10^{-5}$ \\
\hline
\end{tabular}

Tab. 1. $\Delta(z)$ gemäß Gl. (24).

Für $m(T)=M(T) / M(0)$ ergibt sich unter Beachtung aller obigen Abkürzungen

$$
m(T)=1-\frac{3}{2} z^{-3 / 2} \eta(z) .
$$

Wir können demnach zunächst $m(z)$ bestimmen und dann mit Hilfe der Bedeutung von $z$ auf $m(T)$ umrechnen; es ist ja

$$
z= \begin{cases}\frac{2 \pi^{2} \sigma J}{k T} & \text { im Falle c, } \\ \frac{2 \pi^{2} \sigma J}{k T} \frac{1}{2}(1+m(T)) & \text { im Falle d. }\end{cases}
$$

Die auf diesem Wege erhaltenen Funktionen $m(T)$ sind als Kurven $\mathrm{c}$ und $\mathrm{d}$ in Abb. 2 eingetragen; zum Vergleich haben wir noch die experimentelle Kurve für Ni hinzugefügt (e). In Abb. 3 schließlich ist der Tieftemperaturbereich genauer dargestellt, um die Abweichung der Kurven b und d vom $T^{3 / 2-G e s e t z ~ a ~ d e u t-~}$ licher zu zeigen.

\section{§3. Diskussion der Ergebnisse}

Zunächst muß man feststellen, daß das Abbrechen des Energiespektrums nicht sehr wesentlich für den Verlauf von $m(T)$ ist. Viel entscheidender ist offenbar die Temperaturabhängigkeit des Energiespektrums vermittels $f_{0}^{2}$. Man sieht, daß die Berücksichtigung dieses Umstandes die theoretische Funktion $m(T)$ der experimentellen wesentlich ähnlicher macht. Dieser Erfolg berechtigt uns wohl zu der Feststellung, daß unsere Näherung, $f_{l}^{2}$ als Zahlen, nicht als Operatoren aufzufassen und Schwankungen zu vernachlässigen, wirklich vernünftig ist, auf jeden Fall aber eine bedeutend 
bessere Näherung an die Wirklichkeit darstellt als die frühere Annahme $f_{l}^{2}=1$.

Den verbleibenden Unterschied in den $m(T)$ Kurven darf man wohl nicht allein der genäherten Bestimmung der Eigenwerte von $H_{\mathrm{A}}$ und den idealisierten $g(\varepsilon)$ zur Last legen ${ }^{9}$. Vielmehr ist Verf. der Meinung, daß eine Temperaturabhängigkeit des Austauschintegrales, wie sie wegen der thermischen Gitterveränderungen natürlich zu erwarten ist, von uns aber bisher nicht berücksichtigt wurde, $m(T)$ wesentlich verändern kann. Daß schon eine geringe Änderung von $J$ mit $T m(T)$ merklich beeinflußt,

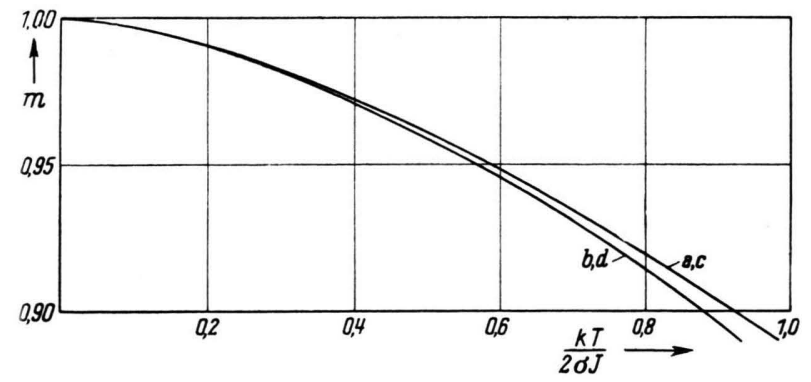

Abb. 3. Tieftemperatur-Verhalten der spontanen Magnetisierung $m$. Theoretische Gesetze.

sieht man am Vergleich unserer Fälle a und b: Man kann ja b auffassen als das $m(T)$ einer Substanz, in welcher das Austauschintegral gleich $f_{0}{ }^{2} J$ ist, also zwischen $T=0$ und $T=T_{\mathrm{c}}$ von $J$ nach $1 / 2 J$ variiert, während diese Größe in a temperaturabhängig bleibt. In beiden Fällen aber sind die Näherungen der alten Spinwellentheorie zugrunde gelegt.

9 Sicherlich ist auch der Einfluß der verschiedenen Gitter-Struktur von untergeordneter Bedeutung.
Aus Abb. 3 ersieht man, daß bei tiefen Temperaturen das Abbrechen des Spektrums $g(\varepsilon)$ sich im Rahmen unserer Rechengenauigkeit überhaupt nicht bemerkbar macht. Dagegen ist es schon von Bedeutung, ob man die Temperaturabhängigkeit von $g$ beachtet oder nicht. Der Abstand $\Delta m$ der beiden Kurven voneinander ist nach Abb. $3 \mathrm{im}$ Gebiet $\frac{k T}{2 \sigma J} \leqq 0,9: \Delta m \leqq 0,01$; d. h. in diesem Gebiete ist der relative Fehler, den man durch die Näherung $f_{l}^{2}=1$ in die Ermittlung von $m(T)$ trägt, maximal von der Größenordnung $1 \%$. Da aber der Curie-Punkt $T_{\mathrm{c}}$ nach Abb. $2 \operatorname{durch} \frac{k T_{\mathrm{c}}}{2 \sigma J}=2,24$ gekennzeichnet ist, hieße dies, daß bis $\mathrm{zu}^{1 / 3}$ der Curie-Temperatur der relative Fehler der Spinwellentheorie in $m(T)$ sicher kleiner als $1 \%$ bleibt. Dabei ist allerdings als ,,richtiges" $m(T)$-Gesetz das unserer Näherungen b oder d benutzt worden. Verf. ist der Meinung, daß dies auch vernünftig ist, solange die Temperaturabhängigkeit des Austauschintegrales $J$ unwesentlich ist. Leider fehlen irgendwelche Abschätzungen dieses Effektes noch vollkommen.

W ir sind damit zum Ausgangspunkte unserer Überlegungen zurückgekehrt und können feststellen, daß die alte Blochsche Spinwellentheorie eine vernünftige Näherung bis zu etwa $1 / 3$ der CurieTemperatur darzustellen scheint und daß eine Erweiterung ihrer Überlegungen auf höhere Temperaturen ohne allzugroßen Aufwand möglich ist, allerdings immer unter der Voraussetzung, daß das Austauschintegral $J$ genähert als temperaturunabhängig angesehen werden darf.

Vielleicht ist die abschließende Bemerkung noch von Interesse, daß nämlich der aus unseren Näherungen $b$ und $\mathrm{d}$ folgende Wert $\frac{k T_{\mathrm{c}}}{2 \sigma J}=2,24$ wesentlich besser zu den aus anderen Theorien für höhere Temperaturen folgenden Werten dieses Verhältnisses paßt als der aus der älteren Näherung a folgende Wert $\frac{k T_{\mathrm{c}}}{2 \sigma J}=4,31$. 